\title{
Neural-Fuzzy-Based Adaptive Sliding Mode Automatic Steering Control of Vision-based Unmanned Electric Vehicles
}

\author{
Jinghua Guo ${ }^{1,2^{*}} \mathbb{D}$, Keqiang Li ${ }^{2}$, Jingjing Fan ${ }^{3}$, Yugong Luo ${ }^{2}$ and Jingyao Wang ${ }^{1}$
}

\begin{abstract}
This paper presents a novel neural-fuzzy-based adaptive sliding mode automatic steering control strategy to improve the driving performance of vision-based unmanned electric vehicles with time-varying and uncertain parameters. Primarily, the kinematic and dynamic models which accurately express the steering behaviors of vehicles are constructed, and in which the relationship between the look-ahead time and vehicle velocity is revealed. Then, in order to overcome the external disturbances, parametric uncertainties and time-varying features of vehicles, a neural-fuzzybased adaptive sliding mode automatic steering controller is proposed to supervise the lateral dynamic behavior of unmanned electric vehicles, which includes an equivalent control law and an adaptive variable structure control law. In this novel automatic steering control system of vehicles, a neural network system is utilized for approximating the switching control gain of variable structure control law, and a fuzzy inference system is presented to adjust the thickness of boundary layer in real-time. The stability of closed-loop neural-fuzzy-based adaptive sliding mode automatic steering control system is proven using the Lyapunov theory. Finally, the results illustrate that the presented control scheme has the excellent properties in term of error convergence and robustness.
\end{abstract}

Keywords: Vision-based unmanned electric vehicles, Automatic steering, Neural-fuzzy adaptive sliding control, Vehicle lateral dynamics

\section{Introduction}

Unmanned electric vehicles have attracted considerable research interests due to its strong power to sufficiently handle these severe problems of traffic congestion and safety. Automatic steering control is devoted to rapidly follow the planned path of automated vehicles, which is regarded as the fundamental issue in the design of automated vehicle systems.

Because automated distributed electric vehicle is a typical nonlinear and time varying system, which possess the properties of parametric uncertainties and strong coupling [1], the research of automatic steering control

\footnotetext{
*Correspondence: guojing_0701@live.cn

${ }^{1}$ School of Aerospace Engineering, Xiamen University, Xiamen 361005, China

Full list of author information is available at the end of the article
}

system is reviewed as an extremely challenging work. Besides, the inevitable external disturbances under the driving conditions greatly enhance the difficulty of the vehicle automatic steering control. Furthermore, the large steady-state errors for automatic steering control of unmanned electric vehicles can be easily caused by the large or changing curvature of the reference trajectory in the highway.

Recently, a lot of automatic steering control strategies are proposed for automated vehicles. A nested PID automatic steering control system of vehicle is established in Ref. [2], and the results illustrate that this strategy has the strong robustness to the uncertain vehicle physical parameters. An intelligent automatic steering control system is presented for automated vehicles, and the parameters of this steering control system are regulated by a novel learning algorithm, the results manifest the 
efficiency of this presented control method [3]. A gain scheduling feedback automatic steering control strategy is presented to ensure the stability of vehicles, in addition, the gains are adaptively adjusted via the fuzzy control technique [4]. An optimal steering control strategy is presented to enhance the driving property of automated vehicle [5], the effectiveness of this genetic optimized fuzzy steering system is proven. In the 2005 grand challenge organized by DARPA, a novel nonlinear automatic steering controller is developed for the robot "Stanley" [6]. A mixed $\mathrm{H}_{2} / \mathrm{H}_{\infty}$ steering controller is proposed and installed in the autonomous snow blower [7]. A hierarchical structure that can emulate a human driver's behavior is designed and the tracking performance of this control method is discussed in Ref. [8]. In order to acquire a time-invariant steering controller, the time-varying terms in the vehicle dynamic model are canceled out by the feedback linearization method [9], an H-infinity optimal automatic steering controller is designed for automated vehicles [10]. Besides, an automatic steering controller is presented for computing the expected wheel steering angle of automated vehicles via the model predictive technique [11-13], the computational complexity and the automatic steering performances of this proposed control scheme are discussed. A novel input/output hybrid steering assistance system is designed for helping the human avert unconscious lane departure [14].

Since the sliding mode control (SMC) method possesses the ability for overcoming the nonlinear behavior, external disturbance and model uncertainty of system, it was adopted to solve the problems of uncertainties and parametric variation of vehicles $[15,16]$, and the results manifest that the SMC method has good dynamic performances at the cost of strong and discontinuous control signals. Nevertheless, because the chattering phenomenon can be caused for its fast switching on the control input [17], SMC method is hard to carry out on an unknown nonlinear dynamic system with uncertain parameters and has limited usage in practice. Integral SMC or SMC with continuous twisting algorithm is usually applied to reduce the chattering $[17,18]$. But, the integral of initial deviation of integral SMC method can lengthen the adjusting time, and deteriorate the transient performance and the stability of system. SMC with continuous twisting algorithm is difficult to balance the antidisturbance capacity and chattering.

The boundary layer strategy is proposed to alleviate the control discontinuity and reduce the chattering [19]. Nevertheless, the system dynamic property will be degraded via adding the thickness of boundary layer. To effectively reduce the model-based demand and wipe off the chattering phenomenon, the applications of artificial neural network or fuzzy theory are proposed to address the chattering reduction problem [20-22]. For example, an adaptive fuzzy SMC method is established for the remotely operated underwater vehicles [23]. To reduce the demand of particular model information and handle the chattering effect, a neural-based SMC control strategy is proposed [24-26]. These applications have been verified as a powerful and efficient way for a nonlinear and uncertain system.

This paper focus on neural-fuzzy-based adaptive sliding mode automatic steering control strategy for the uncertain vehicle dynamic system, which can effectively accomplish the automatic steering control behavior and improve the robust dynamic performance. In the proposed automatic steering control system of unmanned electric vehicles, the control gain of proposed control scheme is regulated by the neural network technique to enhance the tracking performance, and the thickness of boundary layer is adaptively adjusted by the fuzzy theory to relieve the chattering phenomenon.

The main contributions of this work are summarized:

1) A neural-fuzzy-based adaptive sliding mode automatic steering controller is constructed to overcome the uncertain parameters and external disturbances of vehicles, which can guarantee the vision-based unmanned electric vehicles follow the expected path in real-time.

2) In the proposed control system, the control gain of automatic steering control law is adaptively regulated by a radial basis function (RBF) neural network algorithm, and the adaptive thickness of boundary layer is designed via a fuzzy inference, which follows the principles that when the absolute value of sliding surface tends toward zero, the thickness is decreased gradually.

3) Using the Lyapunov theorem, the stability of the closed-loop automatic steering control system is proven.

The remainder of this work is organized as follows. Section 2 shows an uncertain automatic steering dynamic model of vehicles. Section 3 presents a neural-fuzzy-based adaptive sliding mode automatic steering control law to deal with the time-varying and uncertain features of unmanned electric vehicles. Section 4 evaluates the property of the presented control strategy. Section 5 provides the conclusion.

\section{Problem Formulation}

\subsection{Kinematic Model}

The automatic steering kinematic model of vehicle is illustrated in Figure 1, $y_{L}$ denotes the lateral error, it is the distance between the vehicle lateral position and 


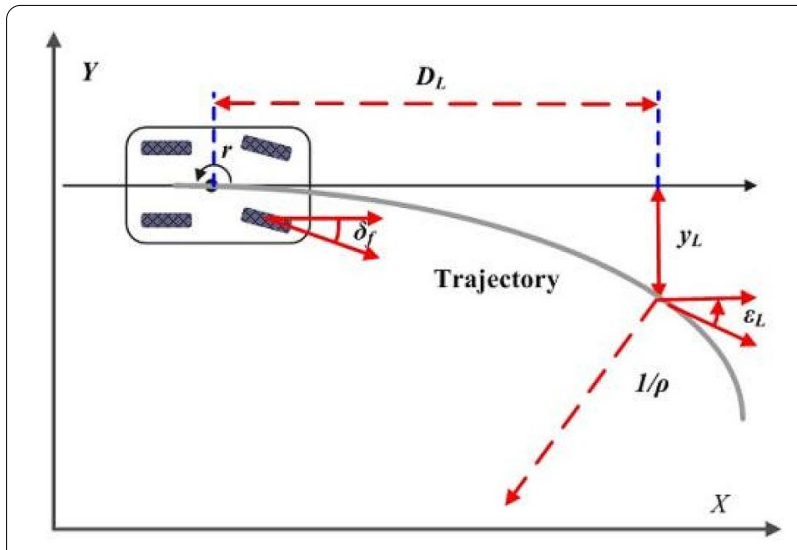

Figure 1 Kinematics model of automated electric vehicle

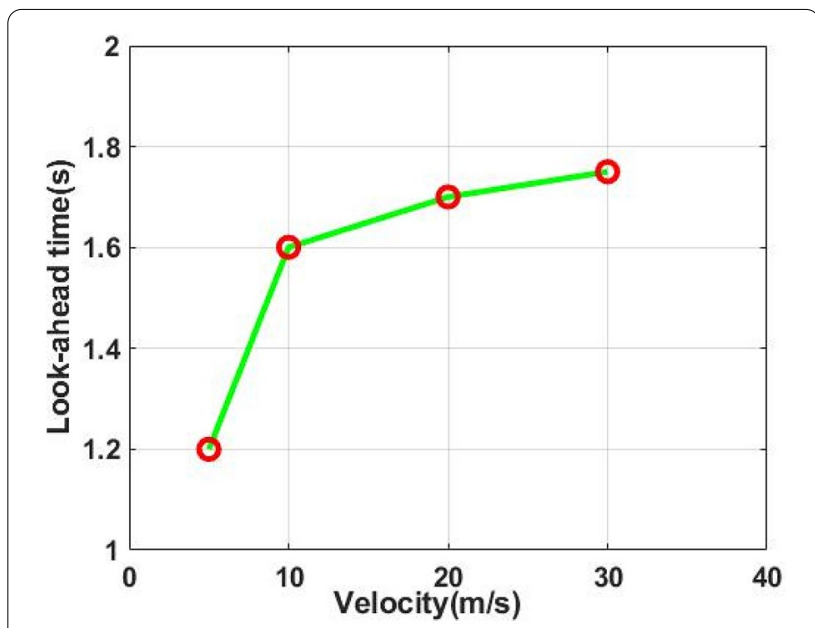

Figure 2 Mapping between the velocity and look-ahead time

smaller, and the look-ahead time remains unchanged when the velocity is increased to a certain value.

\subsection{Vehicle Dynamic Model}

Based on the assumption that the roll, pitch and vertical motions of the unmanned electric vehicles are neglected and the longitudinal velocity $v_{x}$ is set as a constant, then, the decoupled lateral vehicle dynamics model can be deduced as [28]

$$
\left\{\begin{array}{l}
I_{z} \dot{r}=l_{f} F_{y f}-l_{r} F_{y r}, \\
m \dot{v}_{y}=F_{y f}+F_{y r},
\end{array}\right.
$$

In general, the assignment of control problem in Eq. (1) is to develop the automated steering controller to ensure the lateral and angular errors asymptotically converge to the equilibrium point.

The look-ahead distance $D_{L}$ is designed as a saturation function, as follows [5, 6]:

$$
D_{L}= \begin{cases}D_{L \min }, & v_{x}<v_{\min }, \\ v_{x} \cdot t_{1}, & v_{\min } \leq v_{x} \leq v_{\max }, \\ D_{L \max }, & v_{x}>v_{\max },\end{cases}
$$

where $t_{1}$ is the look-ahead time. $D_{L \min }$ and $D_{L \max }$ represent the minimum and maximum values of $D_{L}$, respectively, $v_{\min }$ and $v_{\max }$ represent the minimum and maximum longitudinal velocity values, respectively.

Figure 2 shows the mapping curve between the vehicle longitudinal velocity and the look-ahead time [5, 27]. It can be found that the look-ahead time grows with the increase of vehicle longitudinal velocity. In the low-velocity zone, the slope of increasement of look-ahead time is larger. However, in the high-velocity zone, the slope of increasement of look-ahead time is where $I_{z}$ is the yaw inertia of vehicle, $m$ is the mass of the vehicle, $l_{f}$ represents the distance from the front axle to the mass centre of vehicle, $l_{r}$ represent the distance from the rear axle to the mass centre of vehicle. The front tire cornering stiffness is denoted as $C_{f}$ and the rear tire cornering stiffness is denoted as $C_{r}$, the front wheel steering angle is denoted as $\delta_{f}$. Under the assumptions that the small slip angle is occurred [28], then, the front and rear lateral tire forces $F_{y f}$ and $F_{y r}$ are written as

$$
F_{y f}=C_{f} a_{f}, F_{y r}=C_{r} a_{r} .
$$

In Eq. (4), the front and rear tire sideslip angles $a_{f}$ and $a_{r}$ can be derived as

$$
\begin{aligned}
& \alpha_{f}=\delta_{f}-\tan ^{-1}\left(\frac{v_{y}+l_{f} \dot{\varphi}}{v_{x}}\right), \\
& \alpha_{r}=-\tan ^{-1}\left(\frac{v_{y}-l_{r} \dot{\varphi}}{v_{x}}\right) .
\end{aligned}
$$

Combining Eq. (4) with Eqs. (3) and (5), the following dynamic model is derived as 


$$
\left\{\begin{array}{l}
\dot{v}_{y}=a_{11} v_{y}+a_{12} r+b_{11} \delta_{f}, \\
\dot{r}=a_{21} v_{y}+a_{22} r+b_{21} \delta_{f},
\end{array}\right.
$$

with

$$
\begin{aligned}
& a_{11}=\frac{-\left(C_{r}+C_{f}\right)}{m v_{x}}, \quad a_{12}=\frac{\left(l_{r} C_{r}-C_{f} l_{f}\right)}{m v_{x}}-v_{x} . \\
& a_{21}=\frac{\left(l_{r} C_{r}-l_{f} C_{f}\right)}{I_{z} v_{x}}, \quad a_{22}=\frac{-\left(l_{f}^{2} C_{f}+l_{r}^{2} C_{r}\right)}{I_{z} v_{x}} . \\
& b_{11}=\frac{C_{f}}{m}, \quad b_{21}=\frac{l_{f} C_{f}}{I_{z}} .
\end{aligned}
$$

In Eq. (6), $C_{f}$ and $C_{r}$ are constantly varying because of the variations of the vehicle states and the road conditions, which is represented as

$$
C_{f}=C_{0 f}\left(1+\Delta_{f} \lambda_{f}\right), C_{r}=C_{0 r}\left(1+\Delta_{r} \lambda_{r}\right),
$$

where $\Delta_{f}$ represents the deviation magnitude of front tire cornering stiffness, and $\Delta_{r}$ represents the deviation magnitude of rear tire cornering stiffness. $\lambda_{f}$ and $\lambda_{r}$ denote the time-varying coefficients, they are satisfied as $\left|\lambda_{f}\right| \leq 1$ and $\left|\lambda_{r}\right| \leq 1$, respectively. $C_{0 f}$ denotes the nominal value of $C_{f}, C_{0 r}$ denotes the nominal values of $C_{r}$. In this paper, the deviation magnitudes of tire cornering stiffness are given as $\Delta_{f}=\Delta_{r}=0.6$.

Combining Eqs. (1), (6) and (7), the second derivative of relative position errors $y_{L}$ and $\varepsilon_{L}$ is derived as

$$
\ddot{\boldsymbol{v}}=(\boldsymbol{A}+\Delta \boldsymbol{A}) \dot{\boldsymbol{v}}+(\boldsymbol{E}+\Delta \boldsymbol{E}) \boldsymbol{v}+(\boldsymbol{B}+\Delta \boldsymbol{B}) \boldsymbol{u}+\boldsymbol{d}(t),
$$

with

$$
\boldsymbol{A}=\left[\begin{array}{cc}
0 & A_{1} \\
A_{2} & A_{3}
\end{array}\right], \boldsymbol{E}=\left[\begin{array}{cc}
E_{1} & E_{2} \\
0 & E_{3}
\end{array}\right], \boldsymbol{B}=\left[\begin{array}{l}
B_{1} \\
B_{2}
\end{array}\right], \boldsymbol{d}=\left[\begin{array}{l}
d_{1} \\
d_{2}
\end{array}\right],
$$

and

$$
\begin{aligned}
& A_{1}=\left(v_{x}-a_{11} D_{L}+a_{12}-a_{21} D_{L}^{2}+a_{22} D_{L}\right), \\
& A_{2}=a_{21}, A_{3}=\left(a_{22}-a_{21} D_{L}\right), \\
& E_{1}=\left(a_{11}+a_{21} D_{L}\right), E_{2}=\left(-a_{11} v_{x}-a_{21} v_{x} D_{L}\right), \\
& E_{3}=-a_{21} v_{x}, B_{1}=\left(-b_{11}-b_{21} D_{L}\right), B_{2}=-b_{21}, \\
& d_{1}=\left(a_{11} D_{L}-a_{12}+a_{21} D_{L}^{2}-a_{22} D_{L}\right) v_{x} \rho, \\
& d_{2}=\left(a_{21} D_{L}-a_{22}\right) v_{x} \rho+v_{x} \dot{\rho},
\end{aligned}
$$

where $\boldsymbol{v}=\left[\begin{array}{ll}y_{L} & \varepsilon_{L}\end{array}\right]^{\mathrm{T}}$ represents the state vectors, $\boldsymbol{u}=\delta_{f}$ represents the control input, $\boldsymbol{d}(t)$ represents the external disturbance, $\Delta \boldsymbol{A}, \Delta \boldsymbol{B}$ and $\Delta \boldsymbol{E}$ are the uncertain terms of $\boldsymbol{A}, \boldsymbol{B}$ and $\boldsymbol{E}$.

Assumption 1 The external disturbances and parametric uncertainties in the automatic steering dynamic model of unmanned electric vehicles (8) are bounded as $\Delta \boldsymbol{A}_{l} \leq|\Delta \boldsymbol{A}| \leq \Delta \boldsymbol{A}_{\boldsymbol{h}}, \Delta \boldsymbol{B}_{\boldsymbol{l}} \leq|\Delta \boldsymbol{B}| \leq \Delta \boldsymbol{B}_{\boldsymbol{h}}$ and $\Delta \boldsymbol{E}_{\boldsymbol{l}} \leq|\Delta \boldsymbol{E}| \leq \Delta \boldsymbol{E}_{\boldsymbol{h}}$.
In Eq. (8), $v_{x}$ and $1 / v_{x}$ are time varying, they are varied within $\left[v_{x \min }, v_{x \max }\right]$ and $\left[1 / v_{x \min }, 1 / v_{x \max }\right]$, respectively. $v_{x \min }$ represent the minimum value of longitudinal velocity, and $v_{x \max }$ represent the maximum value of longitudinal velocity. In this paper, the time-varying parameters $v_{x}$ and $1 / v_{x}$ are obtained as $[1,29]$

$$
v_{x}=\sum_{i=1}^{2} \hat{\rho}_{i}(t) \hat{\theta}_{i}, \quad 1 / v_{x}=\sum_{i=1}^{2} \tilde{\rho}_{i}(t) \tilde{\theta}_{i},
$$

where $\hat{\theta}_{i}$ and $\tilde{\theta}_{i}$ denotes the coordinates of vertices, which are given as

$$
\begin{aligned}
& \hat{\theta}_{1}=v_{x \min }, \quad \hat{\theta}_{2}=v_{x \max }, \\
& \tilde{\theta}_{1}=1 / v_{x \max }, \tilde{\theta}_{2}=1 / v_{x \min } .
\end{aligned}
$$

In Eq. (9), $\hat{\rho}_{i}$ and $\tilde{\rho}_{i}$ are the weighting factors, they are expressed as

$$
\begin{aligned}
& \hat{\rho}_{1}(t)=\frac{v_{x \max }-v_{x}}{v_{x \max }-v_{x \min }}, \quad \hat{\rho}_{2}(t)=\frac{v_{x}-v_{x \min }}{v_{x \max }-v_{x \min }}, \\
& \tilde{\rho}_{1}(t)=\frac{1 / v_{x \max }-1 / v_{x}}{1 / v_{x \max }-1 / v_{x \min }}, \quad \tilde{\rho}_{2}(t)=\frac{1 / v_{x}-1 / v_{x \min }}{1 / v_{x \max }-1 / v_{x \min }} .
\end{aligned}
$$

The state-space model in Eq. (8) can be approximated as

$$
\begin{aligned}
\ddot{v}(t)= & \sum_{i=1}^{4} \rho_{i}(t)\left[\left(A_{i}+\Delta A_{i}\right) \dot{v}(t)+\left(E_{i}+\Delta E_{i}\right) v(t)\right. \\
& +(B+\Delta B) u(t)+d(t)] \\
& =(A(\rho)+\Delta A(\rho)) \dot{v}(t)+(E(\rho)+\Delta E(\rho)) v(t) \\
& +(B+\Delta B) u(t)+d(t),
\end{aligned}
$$

with

$$
\begin{gathered}
\rho_{1}=\hat{\rho}_{1} \tilde{\rho}_{1}, \quad \rho_{2}=\hat{\rho}_{1} \tilde{\rho}_{2}, \quad \rho_{3}=\hat{\rho}_{2} \tilde{\rho}_{1}, \quad \rho_{4}=\hat{\rho}_{2} \tilde{\rho}_{2} \\
\boldsymbol{\rho}=\left[\begin{array}{llll}
\rho_{1} & \rho_{2} & \rho_{3} & \rho_{4}
\end{array}\right]^{\mathrm{T}} .
\end{gathered}
$$

The designed state-space model (14) can describe the parametric uncertainties, external disturbances and time-varying features of unmanned electric vehicles.

\section{Neural-Fuzzy-based Adaptive Sliding Mode Automatic Steering Control Strategy}

The mission of automatic steering control system is to ensure that the unmanned electric vehicles accurately track the expected road in a continuous and slippy way, while improving the stability of vehicles $[1$, 30]. However, unmanned electric vehicles possess the 
properties of time-varying, external disturbances and parametric uncertainties, the proposed automatic steering control strategy should availably conquer these dynamic characteristics.

As shown in Figure 3, a neural fuzzy based adaptive sliding mode automatic steering control strategy of unmanned electric vehicles is proposed to supervise the lateral dynamics of vehicles. In this architecture, a new sliding surface is designed, then, the adaptive control gain (ACG) of variable structure control law is approximated via the neural network system, in real-time, and the variable boundary layer (ABL) is introduced and adaptively regulating by the fuzzy theory. This novel adaptive sliding mode controller can guarantee the stability of closedloop automatic steering control system of vehicles.

\subsection{Traditional SMC Controller}

It is well known that the basic idea of SMC is to force its movement in the sliding mode surface, therefore, the construction of sliding surface is a crucial part of SMC to achieve the desired control specifications and performances. A novel proportional-integral-differential sliding surface is designed as

$$
\boldsymbol{s}(t)=\boldsymbol{k}_{p} \boldsymbol{e}(t)+\boldsymbol{k}_{i} \int \boldsymbol{e}(t) \mathrm{d} t+\boldsymbol{k}_{\boldsymbol{d}} \frac{\mathrm{d}}{\mathrm{d} t} \boldsymbol{e}(t),
$$

where $\boldsymbol{k}_{\boldsymbol{p}}$ is the positive proportional gain matrix, $\boldsymbol{k}_{\boldsymbol{i}}$ is the positive integral gain matrix, and $\boldsymbol{k}_{\boldsymbol{d}}$ is the positive derivative gain matrix. $\boldsymbol{e}(t)=\boldsymbol{v}(t)-\boldsymbol{v}_{\boldsymbol{d}}(t)$ denotes the tracking error, and $\boldsymbol{v}_{\boldsymbol{d}}(t)=0, \boldsymbol{s}=\left[s_{1}, s_{2}\right]$.

The time derivative of the sliding surface (16) can be obtained as

$$
\begin{aligned}
\dot{\boldsymbol{s}}(t)= & \boldsymbol{k}_{d} \ddot{\boldsymbol{e}}(t)+\boldsymbol{k}_{p} \dot{\boldsymbol{e}}(t)+\boldsymbol{k}_{i} \boldsymbol{e}(t) \\
= & \boldsymbol{k}_{d}((\boldsymbol{A}+\Delta \boldsymbol{A}) \dot{v}(t)+(\boldsymbol{E}+\Delta \boldsymbol{E}) v+(\boldsymbol{B}+\Delta \boldsymbol{B}) \boldsymbol{u}+\boldsymbol{d}(t)) \\
& +\boldsymbol{k}_{p} \dot{v}(t)+\boldsymbol{k}_{i} v(t) \\
= & \left(\boldsymbol{k}_{d}(\boldsymbol{A}+\Delta \boldsymbol{A})+\boldsymbol{k}_{p}\right) \dot{v}(t)+\left(\boldsymbol{k}_{d}(\boldsymbol{E}+\Delta \boldsymbol{E})+\boldsymbol{k}_{i}\right) v(t) \\
& +\boldsymbol{k}_{d}(\boldsymbol{B}+\Delta \boldsymbol{B}) u+\boldsymbol{k}_{d} \boldsymbol{d}(t)
\end{aligned}
$$

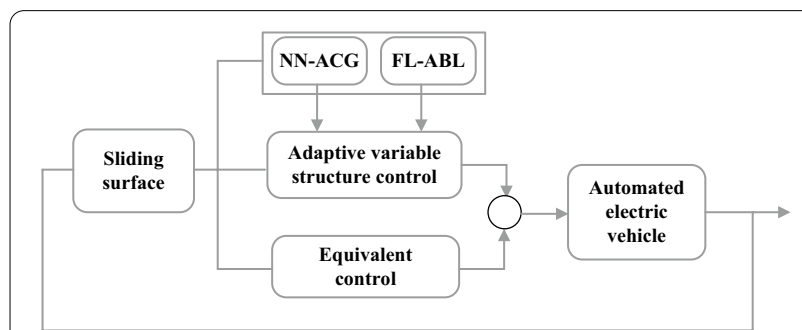

Figure 3 Neural-fuzzy-based adaptive sliding mode automatic steering control architecture
Setting Eq. (17) to zero as

$$
\left.\dot{\boldsymbol{s}}(t)\right|_{\boldsymbol{u}(t)=\boldsymbol{u}_{\boldsymbol{e q}}(t)}=0 .
$$

The external disturbance and uncertain terms is ignored, and the equivalent control law is derived as

$$
\boldsymbol{u}_{e q}(t)=-\left(\boldsymbol{k}_{\boldsymbol{d}} \boldsymbol{B}\right)^{-1}\left(\left(\boldsymbol{k}_{\boldsymbol{d}} \boldsymbol{A}+\boldsymbol{k}_{\boldsymbol{p}}\right) \dot{\boldsymbol{v}}+\left(\boldsymbol{k}_{\boldsymbol{d}} \boldsymbol{E}+\boldsymbol{k}_{\boldsymbol{i}}\right) \boldsymbol{v}\right) .
$$

Equivalent control term is invalid when the tracking errors are away from the sliding surface. Hence, to direct the system errors to the sliding surface, an additional variable structure control term can be designed as

$$
\boldsymbol{u}_{r}(t)=-\boldsymbol{K}(t) \operatorname{sign}(s(t)),
$$

where $\boldsymbol{K}(t)$ denotes the control gain, the sign function $\operatorname{sign}(\cdot)$ can be written as

$$
\operatorname{sign}(s(t))= \begin{cases}1, & \text { if } \mathrm{s}(t)>0 \\ 0, & \text { if } \mathrm{s}(t)=0 \\ -1, & \text { if } \mathrm{s}(t)<0\end{cases}
$$

Combining Eqs. (19) and (20), hence, the total automatic steering control law

$$
u=u_{e q}+u_{r} .
$$

Remark 1 The control gain $K(t)$ is involved with the bound of parametric uncertainties, which is hard to be acquired precisely in practical use. Hence, the larger control gain will produce the grievous chattering and excite unstable system dynamics.

\subsection{NN-based Adaptive Control Gain}

The magnitude of external disturbances, parametric uncertainties and signal noises has a vital impact on the control gain $K(t)$. For purpose of dealing with this problem, an adaptive control gain scheme using the neural network (NN) technique is proposed in this subsection. Specifically, the switching control gain $K(t)$ of variable structure control term is estimated by a neural network inference system to alleviate the chattering and enhance the dynamic property of the automatic steering controller.

Definition 1 Let $S$ be a compact simply connected set of $\boldsymbol{R}^{n}$. In general, for a continuous function $\boldsymbol{f}(x)$, there exists an adjustable weight vector $\boldsymbol{R}$ and a radial basis 
Gaussian vector $\boldsymbol{\phi}(x)$ such that $\boldsymbol{f}(x)-\boldsymbol{R}^{\mathrm{T}} \boldsymbol{\phi}(x)$ has a NN functional reconstruction error vector $\varepsilon(x)$.

The optimal weight vector $\boldsymbol{R}^{*}$ is given as

$$
\boldsymbol{R}^{*}=\arg \min \left\{\sup _{x \in S}\left|f(x)-\boldsymbol{R}^{\mathrm{T}} \boldsymbol{\phi}(x)\right|\right\} .
$$

Neural network system has well function approximation capability and fault-tolerance capability at the same time [31, 32]. In this section, to acquire an excellent approximation to the unknown and variable control gain, the control gain $K(t)$ is directly depended on a RBF neural network algorithm, as follows

$$
\hat{\boldsymbol{K}}(t)=\sum_{i=1}^{m} \hat{\boldsymbol{w}}_{i} \boldsymbol{h}_{\boldsymbol{i}}(\boldsymbol{s})=\hat{\boldsymbol{W}}^{\mathrm{T}} \boldsymbol{H}(\boldsymbol{s}),
$$

$\hat{\boldsymbol{W}}=\left[\hat{w}_{1}, \cdots, \hat{w}_{m}\right]^{\mathrm{T}}$ is the adjustable weight vector of RBF, $\boldsymbol{H}(\boldsymbol{s})=\left[h_{1}(\boldsymbol{s}), \cdots, h_{m}(\boldsymbol{s})\right]^{\mathrm{T}}$ is the Gaussian function, which can be in the form as

$$
h_{i}(\boldsymbol{s})=\exp \left(-\frac{\left\|\boldsymbol{s}-c_{i}\right\|}{b_{i}}\right), i=1,2, \cdots, m,
$$

where $c_{i}$ and $b_{i}$ are the parameters of the Gaussian function.

The desired control gain $\boldsymbol{K}^{*}(t)$ is given as

$$
\boldsymbol{K}^{*}(t)=\boldsymbol{W}^{* \mathrm{~T}} \boldsymbol{H}(\boldsymbol{s})+\varepsilon^{\prime},
$$

where $\boldsymbol{\varepsilon}^{\prime}$ is the approximation error, $\boldsymbol{W}^{*}(t)$ is a constant ideal weight matrix, and there exists a position constant $\eta$ which satisfies

$$
\boldsymbol{K}^{*}(t)=\eta
$$

In order to guarantee the asymptotical stability of $s(t) \rightarrow 0$ as $t \rightarrow \infty$, for the controlled system in Eq. (14), an adaptive control law of parameter $\hat{W}$ can be obtained as

$$
\dot{\hat{W}}=r k_{d} B s H(s),
$$

where $r$ is the positive constant, and the weight estimation errors is $\tilde{W}=\hat{W}-W^{*}$.

Remark 2 It is interesting to note that the finite nodes of RBF neural network can lead to a small modeling error $\boldsymbol{\varepsilon}^{\prime}$, but, an RBF neural network can be found such that $\left\|\boldsymbol{\varepsilon}^{\prime}\right\| \leq \boldsymbol{\varepsilon}_{N}$, where $\|\cdot\|$ is the Euclidean norm of a vector, $\boldsymbol{\varepsilon}_{N}$ denotes a positive constant.

Remark 3 It is well known that the adaptive law may cause drift of the tunable parameters in the case of the presence of measurement errors. In order to deal with this drawback, the adaptive law could be updated as $\sigma$ modification technique [33].

\subsection{FL-based Adaptive Boundary Layer}

To further relieve the chattering phenomenon and enhance the dynamic performance, a boundary layer is designed in the variable structure control term, as follows

$$
\boldsymbol{u}_{\boldsymbol{r}}=\hat{\boldsymbol{K}}(t) \operatorname{sat}\left(\frac{\boldsymbol{s}}{\boldsymbol{\Delta}}\right) .
$$

The function $\operatorname{sat}(\cdot)$ represents the actuator saturation. It is interesting to note that the chattering phenomenon can be alleviated, however, the unreasonable saturated parameter vector $\Delta=\left[\Delta_{1}, \Delta_{2}\right]$ can damage the dynamic performance and robustness.

In order to deal with the above problems and achieve the better tracking performance, the adaptive adjusting strategies of thickness of boundary layer are attracting more attention [34, 35]. Here, an adaptive variable boundary layer is designed by the fuzzy logic approach. The input variable is the absolute value of sliding surface $\left|s_{i}\right|$, and the output variable is the thickness $\Delta_{i}$. The membership functions of the linguistic terms very small (VS), small (S), medium (M), large (L), very large $(\mathrm{VL})$ are assigned to the input variable $\left|s_{i}\right|$, and the linguistic terms very narrow (VN), narrow $(\mathrm{N})$, medium $(\mathrm{M})$, wide $(\mathrm{W})$, very wide $(\mathrm{VW})$ are assigned to the output variable $\Delta_{i}$. As illustrated in Figure 4, the input and output variables are fuzzified by the triangular and trapezoidal membership functions.

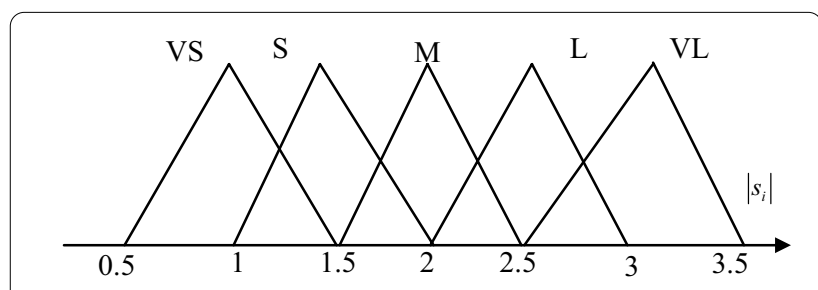

(a) Membership functions of input $\left(\left|s_{i}\right|\right)$

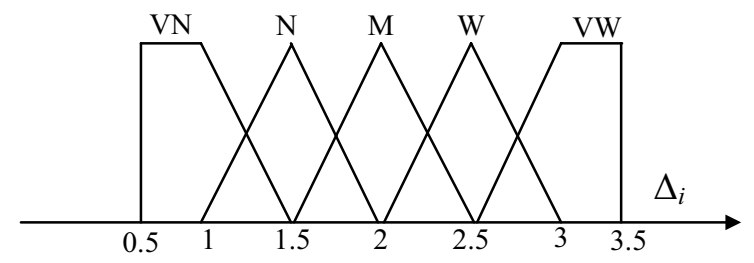

(b) Membership functions of output $\left(\Delta_{i}\right)$

Figure 4 Membership functions 
Fuzzy partitions with triangular membership functions could produce entropy equalization, triangular membership functions are employed to give an errorfree reconstruction in this paper.

When the system states are going to be outside the boundary layer, for realizing the fast convergence to the sliding surface, the thickness of boundary layer should be narrowed. When the system states are within the boundary layer, in order to alleviate the chattering and avoid overshoot, the thickness of boundary layer should be widened. Based on these principles, the linguistic fuzzy rules are expressed as the following procedure:

$R_{1}$ : if $\left|s_{i}\right|$ is very large then $\left|\Delta_{i}\right|$ is very narrow

$R_{2}$ : if $\left|s_{i}\right|$ is large then $\left|\Delta_{i}\right|$ is narrow

$R_{3}$ : if $\left|s_{i}\right|$ is medium then $\left|\Delta_{i}\right|$ is medium

$R_{4}$ : if $\left|s_{i}\right|$ is small then $\left|\Delta_{i}\right|$ is wide

$R_{5}$ : if $\left|s_{i}\right|$ is very small then $\left|\Delta_{i}\right|$ is very wide

The fuzzy rules is designed in accordance with the principle that the thickness of boundary layer is decreased gradually when the vehicle lateral control system tends toward steady state. In the first rule $R_{1}$, if the absolute value of sliding surface $\left|s_{i}\right|$ is very large, then the thickness $\left|\Delta_{i}\right|$ of boundary layer is very narrow. Fuzzy rule $R_{1}$ narrows the thickness of boundary layer to accomplish the goal of achieving the sliding surface rapidly. In the second rule $R_{2}$, if the absolute value of sliding surface $\left|s_{i}\right|$ is large, then the thickness $\left|\Delta_{i}\right|$ of boundary layer is narrow. In the third rule $R_{3}$, if the absolute value of sliding surface $\left|s_{i}\right|$ is medium, then the thickness $\left|\Delta_{i}\right|$ of boundary layer is medium. In the fourth rule $R_{4}$, if the absolute value of sliding surface $\left|s_{i}\right|$ is small, then the thickness $\left|\Delta_{i}\right|$ of boundary layer is wide. In the fifth rule $R_{5}$, if the absolute value of sliding surface $\left|s_{i}\right|$ is very small, then the thickness $\left|\Delta_{i}\right|$ of boundary layer is very wide. Fuzzy rule $R_{5}$ broadens the thickness to accomplish the goal of alleviating the chattering phenomena.

Remark 4 The presented adaptive boundary layer approach follows the principles that when the absolute value of sliding surface $|\boldsymbol{s}|$ tends to zero, the thickness is decreased gradually.

\subsection{Stability Analysis}

Lemma 1 [36] If a scalar function $V(x, t)$ satisfies the following conditions

(i) $\boldsymbol{V}(x, t)$ is lower bounded.

(ii) $\dot{\boldsymbol{V}}(x, t)$ is negative semi-definite. (iii) $\dot{V}(x, t)$ is uniformly continuous in time.

Then, $\dot{V}(x, t) \rightarrow 0$ as $t \rightarrow \infty$.

Lemma 2 [36] (Barbalat) If the differentiable function $f(t)$ has a finite limit as $t \rightarrow \infty$, and iff is uniformly continuous, then $f(t) \rightarrow 0$ as $t \rightarrow \infty$.

Lemma 3 [36] If the vector function $f(x, t)$ has continuous and bounded first partial derivatives with respect to $x$ and $t$, for all $x$ in a ball $B_{r}$ and for all $t \geq 0$, then the equilibrium point at the origin is exponentially stable, if and only if, there exists a function $V(x, t)$ and strictly positive constants $a_{1}, a_{2}, a_{3}, a_{4}$ such that $\forall x \in B_{r}, \forall t \geq 0$ :

$$
\begin{gathered}
a_{1}\|x\|^{2} \leq V(x, t) \leq a_{2}\|x\|^{2}, \\
\dot{\boldsymbol{V}} \leq-a_{3}\|x\|^{2}, \\
\left\|\frac{\partial \boldsymbol{V}}{\partial x}\right\| \leq a_{4}\|x\| .
\end{gathered}
$$

Theorem 1 For the steering system Eq. (14) of automated electric vehicles, if the automatic steering controller is established as Eq. (22) with Eqs. (19) and (29), and the weight adaptive control law is given as Eq. (28), then the tracking errors will converge to zero.

Proof The Lyapunov function candidate is considered as [34]

$$
\boldsymbol{V}(t)=\frac{1}{2} \boldsymbol{s}^{2}+\frac{1}{2 \boldsymbol{r}} \tilde{\boldsymbol{W}}^{T} \tilde{\boldsymbol{W}} .
$$

Owing to the fact that $\tilde{W}=\hat{W}-W^{*}$, then, the derivative of Eq. (31) can be obtained as

$$
\dot{\boldsymbol{V}}(t)=\boldsymbol{s} \dot{\boldsymbol{s}}+\frac{1}{\boldsymbol{r}} \tilde{\boldsymbol{W}}^{T} \dot{\hat{\boldsymbol{W}}} .
$$

Rewriting Eq. (14), yields

$$
\begin{aligned}
\ddot{\boldsymbol{v}} & =\boldsymbol{A} \dot{\boldsymbol{v}}+\boldsymbol{E} \boldsymbol{v}+\boldsymbol{B} \boldsymbol{u}+\Delta \boldsymbol{A} \dot{\boldsymbol{v}}+\Delta \boldsymbol{E} \boldsymbol{v}+\Delta \boldsymbol{B} \boldsymbol{u}+\boldsymbol{d}(t) \\
& =\boldsymbol{A} \dot{\boldsymbol{v}}+\boldsymbol{E} \boldsymbol{v}+\boldsymbol{B} \boldsymbol{u}+\boldsymbol{L}_{\boldsymbol{r}}
\end{aligned}
$$

where $\boldsymbol{L}_{r}=\Delta \boldsymbol{A} \dot{\boldsymbol{v}}+\Delta \boldsymbol{E} \boldsymbol{v}+\Delta \boldsymbol{B} \boldsymbol{u}+\boldsymbol{d}(t)$. Based on the Assumption 1, it is interesting to note that the disturbance $\boldsymbol{L}_{\boldsymbol{r}}$ is bounded.

Substituting Eq. (33) into the function $s \dot{s}$, then

$$
\begin{aligned}
\boldsymbol{s} \dot{\boldsymbol{s}} & =\boldsymbol{s}\left(\boldsymbol{k}_{\boldsymbol{d}} \ddot{\boldsymbol{e}}(t)+\boldsymbol{k}_{p} \dot{\boldsymbol{e}}(t)+\boldsymbol{k}_{i} \boldsymbol{e}(t)\right) \\
& =\boldsymbol{s}\left(\boldsymbol{k}_{\boldsymbol{d}}(\boldsymbol{A}+\Delta \boldsymbol{A}) \dot{\boldsymbol{v}}(t)+\boldsymbol{k}_{\boldsymbol{d}}(\boldsymbol{E}+\Delta \boldsymbol{E}) \boldsymbol{v}(t)\right) \\
& +\boldsymbol{s}\left(\boldsymbol{k}_{\boldsymbol{d}}(\boldsymbol{B}+\Delta \boldsymbol{B}) \boldsymbol{u}+\boldsymbol{k}_{\boldsymbol{d}} \boldsymbol{d}(t)\right)+\boldsymbol{s}\left(\boldsymbol{k}_{p} \dot{\boldsymbol{v}}(t)+\boldsymbol{k}_{i} \boldsymbol{v}(t)\right) \\
& =\boldsymbol{s}\left(\boldsymbol{k}_{\boldsymbol{d}} \boldsymbol{A} \dot{\boldsymbol{v}}(t)+\boldsymbol{k}_{\boldsymbol{d}} \boldsymbol{E v}(t)+\boldsymbol{k}_{\boldsymbol{d}} \boldsymbol{B} \boldsymbol{u}+\boldsymbol{k}_{\boldsymbol{p}} \dot{\boldsymbol{v}}(t)+\boldsymbol{k}_{\boldsymbol{i}} \boldsymbol{v}(t)\right)+\boldsymbol{s} \boldsymbol{k}_{\boldsymbol{d}} \boldsymbol{L}_{\boldsymbol{r}} .
\end{aligned}
$$


Substituting the equivalent control law Eq. (19) and variable structure control term Eqs. (29) to (34), as follows:

$$
\begin{aligned}
& \dot{\boldsymbol{V}}=\boldsymbol{s}\left(\boldsymbol{k}_{\boldsymbol{d}} \boldsymbol{A} \dot{\boldsymbol{v}}(t)+\boldsymbol{k}_{\boldsymbol{d}} \boldsymbol{E} \boldsymbol{v}(t)+\boldsymbol{k}_{\boldsymbol{d}} \boldsymbol{B} \boldsymbol{u}+\boldsymbol{k}_{\boldsymbol{p}} \dot{\boldsymbol{v}}(t)+\boldsymbol{k}_{\boldsymbol{i}} \boldsymbol{v}(t)\right) \\
& +\boldsymbol{s} \boldsymbol{k}_{\boldsymbol{d}} \boldsymbol{L}_{\boldsymbol{r}}+\frac{1}{\boldsymbol{r}} \tilde{\boldsymbol{W}}^{\mathrm{T}} \dot{\hat{\boldsymbol{W}}} \\
& \left.=\boldsymbol{s}\left(\boldsymbol{k}_{\boldsymbol{d}} \boldsymbol{A} \dot{\boldsymbol{v}}(t)\right)+\boldsymbol{k}_{\boldsymbol{d}} \boldsymbol{E} \boldsymbol{v}(t)+\boldsymbol{k}_{\boldsymbol{p}} \dot{\boldsymbol{v}}(t)+\boldsymbol{k}_{\boldsymbol{i}} \boldsymbol{v}(t)\right) \\
& +\boldsymbol{s}\left(\boldsymbol { k } _ { \boldsymbol { d } } \boldsymbol { B } \left(-\left(\boldsymbol{k}_{\boldsymbol{d}} \boldsymbol{B}\right)^{-1}\left(\left(\boldsymbol{k}_{\boldsymbol{d}} \boldsymbol{A}+\boldsymbol{k}_{\boldsymbol{p}}\right) \dot{\boldsymbol{v}}(t)\right.\right.\right. \\
& \left.\left.\left.+\left(\boldsymbol{k}_{\boldsymbol{d}} \boldsymbol{E}+\boldsymbol{k}_{\boldsymbol{i}}\right) \boldsymbol{v}(t)\right)-\hat{\boldsymbol{K}}_{\boldsymbol{r}}(t) \operatorname{sat}(\boldsymbol{s} / \Delta)\right)\right) \\
& +\boldsymbol{s} \boldsymbol{k}_{\boldsymbol{d}} \boldsymbol{L}_{\boldsymbol{r}}+\frac{1}{\boldsymbol{r}} \tilde{\boldsymbol{W}}^{\mathrm{T}} \dot{\hat{\boldsymbol{W}}}
\end{aligned}
$$

then,

$$
\begin{aligned}
& \dot{\boldsymbol{V}} \leq \boldsymbol{s} \boldsymbol{k}_{\boldsymbol{d}} \boldsymbol{B}\left(-\hat{\boldsymbol{K}}(t)+\boldsymbol{K}^{*}(t)-\boldsymbol{K}^{*}(t)\right)+\boldsymbol{s} \boldsymbol{k}_{\boldsymbol{d}} \boldsymbol{L}_{\boldsymbol{r}}+\frac{1}{\boldsymbol{r}} \tilde{\boldsymbol{W}}^{\mathrm{T}} \dot{\hat{\boldsymbol{W}}} \\
& \leq \boldsymbol{s} \boldsymbol{k}_{d} \boldsymbol{B}\left(-\tilde{\boldsymbol{W}}^{\mathrm{T}} \boldsymbol{H}(\boldsymbol{s})+\boldsymbol{\varepsilon}^{\prime}-\eta\right)+\boldsymbol{s} \boldsymbol{k}_{d} \boldsymbol{L}_{\boldsymbol{r}}+\frac{1}{r} \tilde{\boldsymbol{W}}^{\mathrm{T}}\left(\boldsymbol{r} \boldsymbol{s} \boldsymbol{k}_{d} \boldsymbol{B} \boldsymbol{H}(\boldsymbol{s})\right) \\
& \leq s k_{d} B\left(\varepsilon^{\prime}-\eta\right)+s k_{d} L_{r} \\
& \leq-k_{d} B \eta|s|+k_{d} L_{r}|s|+|s| k_{d} B \varepsilon^{\prime} .
\end{aligned}
$$

Selecting $\boldsymbol{B} \boldsymbol{\eta}>\boldsymbol{L}_{\boldsymbol{r}}$, it can be found that

$$
\dot{V}<0 \text {. }
$$

Thus, the proposed control scheme can ensure the asymptotic stability of the closed-loop automatic steering control system.

\section{Performance Verification}

In order to validate the feasibility of the presented adaptive sliding mode control (ASMC) strategy in the aforementioned section, a series of simulation and experiment tests are implemented. In addition, the presented AMSC automatic steering control scheme is contrasted with the MPC controller $[11,12]$ and the linear quadratic regular (LQR) controller. Main parameter values of vehicle model is show in Table 1.

Table 1 Main parameter values of vehicle model

\begin{tabular}{ll}
\hline Parameter & Value \\
\hline Vehicle mass $m(\mathrm{~kg})$ & 1800 \\
Yaw inertia of vehicle $I_{z}\left(\mathrm{~kg} \cdot \mathrm{m}^{2}\right)$ & 2500 \\
Distance from the front axle to the mass centre $I_{f}(\mathrm{~m})$ & 1.03 \\
Distance from the rear axle to the mass centre $I_{r}(\mathrm{~m})$ & 1.49 \\
Nominal cornering stiffness of front tires $C_{0 f}(\mathrm{kN} / \mathrm{rad})$ & 40 \\
Nominal cornering stiffness of rear tires $C_{0 r}(\mathrm{kN} / \mathrm{rad})$ & 40 \\
\hline
\end{tabular}

\subsection{Simulation Test}

Firstly, a Matlab-Adams co-simulation test of unmanned electric vehicles driving on the icy road is carried out, the adhesion coefficient of road is set as 0.2 , the external disturbance and parametric uncertainty are obvious in this severe operation conditions. A quasi-straight line is applied as a desired path to verify the feasibility of this ASMC automatic steering control approach. The longitudinal velocity of vehicle is given as $90 \mathrm{~km} / \mathrm{h}$, the initial value of lateral error is assumed as $0.3 \mathrm{~m}$ and the initial value of angular error is assumed as $-3^{\circ}$.

The compared steady-state response results of vehicle states are demonstrated in Figure 5. Figure 5(a) demonstrates the dynamic response of lateral error, it can be found that the overshoot of lateral error with the ASMC control scheme is smaller than the LQR control method and the MPC control method. The regulation

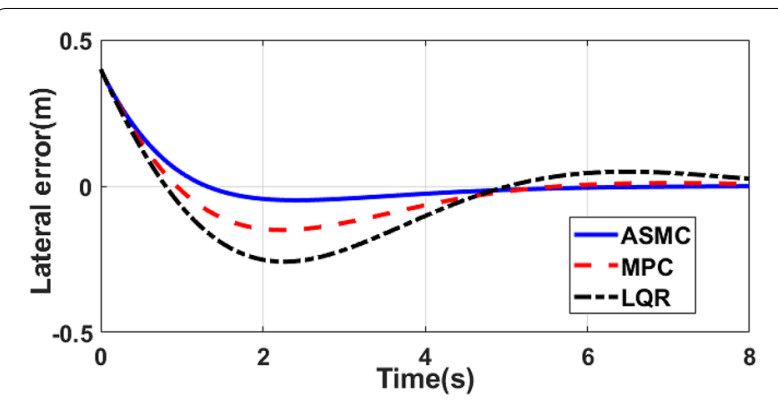

(a)

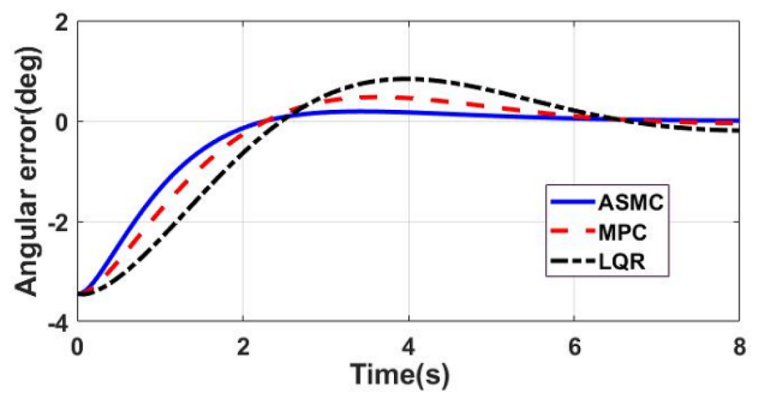

(b)

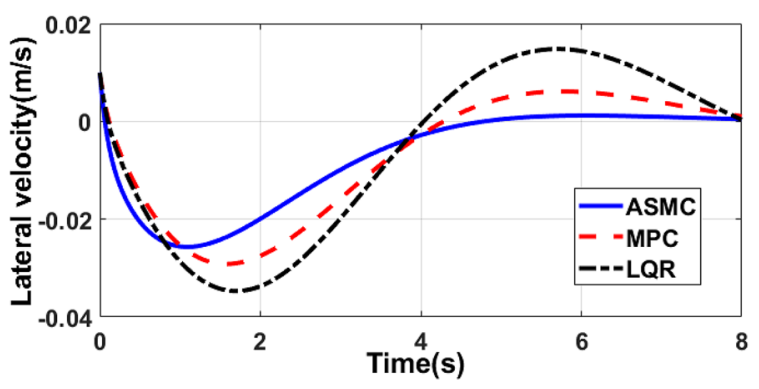

(c)

Figure 5 Vehicle states in simulation test 
time of lateral error with the ASMC controller is $2 \mathrm{~s}$, but the regulation times with the MPC and LQR control methods are $4 \mathrm{~s}$ and $6 \mathrm{~s}$, respectively. Figure 5(b) illustrates the dynamic response of angular error, it can be found that the overshoots of angular error regulated by the presented ASMC control strategy is closed to zero. Figure 5(c) shows the results of lateral velocity, It is interesting to note that the presented ASMC control scheme has the smaller oscillatory behavior, and the regulation time is quicker than the LQR controller and the MPC controller. Figure 5 demonstrates that the presented ASMC approach has the excellent automatic steering property under the abominable circumstances.

\subsection{Experimental Test}

As shown in Figure 6, the prototype automated electric vehicle has the three-layer system architecture and possess the basic functions of environments apperceiving, dynamic decision making and planning, action control and execution [37-39]. The vision system can detect and extract the characteristics of reference path with less than $20 \mathrm{~ms} /$ frame processing time. The sampling period of control system for automated electric vehicle is $100 \mathrm{~Hz}$, and the information of vehicle's attitude and position are acquired by the wheel encoders and GPS receivers.

In addition, the numerical indicators like the integral of absolute errors (IAE) and the integral of time per absolute errors (ITAE) are computed to evaluate the control performance, as follows:

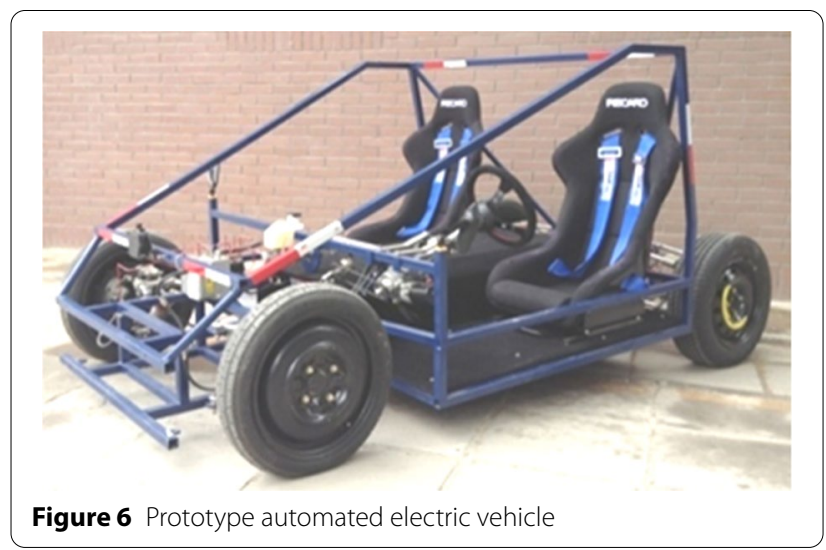

$$
\left\{\begin{array}{l}
I_{I A E}\left(e_{i}(t)\right)=\int_{0}^{T}\left|e_{i}(t)\right| \mathrm{d} t, \\
I_{I T A E}\left(e_{i}(t)\right)=\int_{0}^{T} t \cdot e_{i}(t) \mathrm{d} t,
\end{array} \quad i=1,2,\right.
$$

where $e_{1}(t)=y_{L}$ and $e_{2}(t)=\varepsilon_{L}$.

Two practical trials are implemented so as to investigate the dynamic property of the presented ASMC control strategy, and the variation of road shape is considered for different operation conditions. The profiles of desired road of experimental test I is illustrated in Figure 7, and the vehicle velocity is given as $25 \mathrm{~km} / \mathrm{h}$. The initial lateral and angular errors are $0.3 \mathrm{~m}$ and $-2.9^{\circ}$, respectively.

Figure 8 demonstrates the vehicle states regulated by three controllers in the first experimental test. Figure 8(a) shows the compared dynamic response of lateral errors, it can be found that the lateral errors adjusted by the presented ASMC control method, the MPC control method and LQR control method are within $\pm 0.3 \mathrm{~m}$, $\pm 0.5 \mathrm{~m}$ and $\pm 0.7 \mathrm{~m}$. Figure 8 (b) illustrates the dynamic response of angular errors, and it demonstrates that all the control approaches can limit the angular error within $\pm 5^{\circ}$, but, contrasted to the MPC steering controller and the LQR steering controller, the oscillation and overshoot of angular error adjusted by the presented ASMC steering control scheme is smaller than two other controllers. Figure 8(c) displays the dynamic response of lateral velocity, it is interesting to note that the stability and riding comfort of automated electric vehicle regulated by the proposed ASMC steering control strategy is better than the MPC steering control method. Figure 8(d) displays the control input of front steer angle, it manifests that the magnitudes of the steering angles produced by all the control approaches are basically the same, but the proposed ASMC control approach has the smooth control input. Since the discontinuity of proposed ASMC control

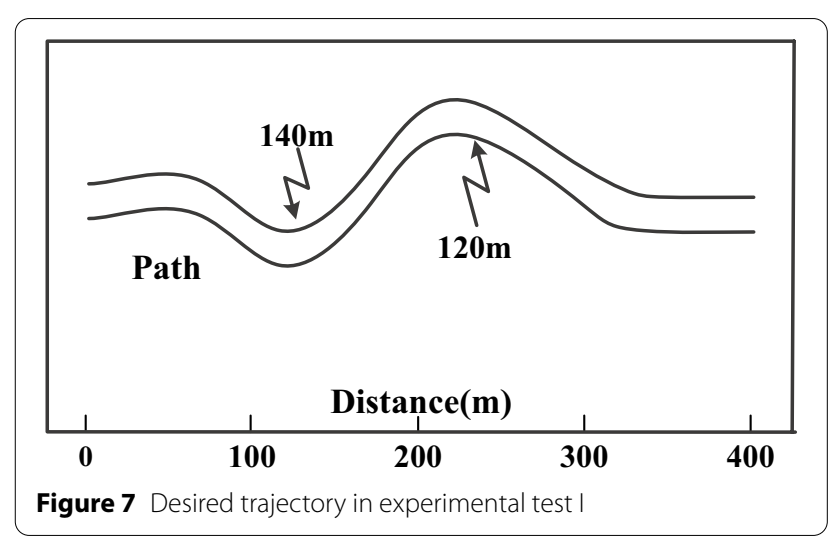




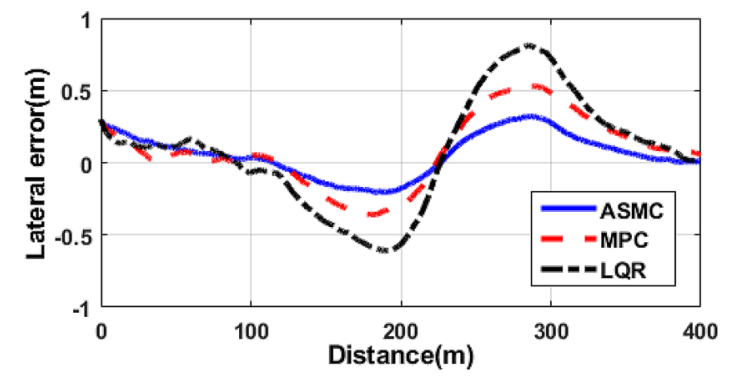

(a)

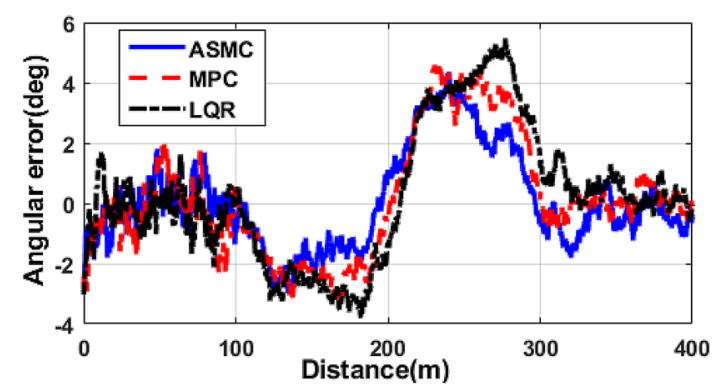

(b)

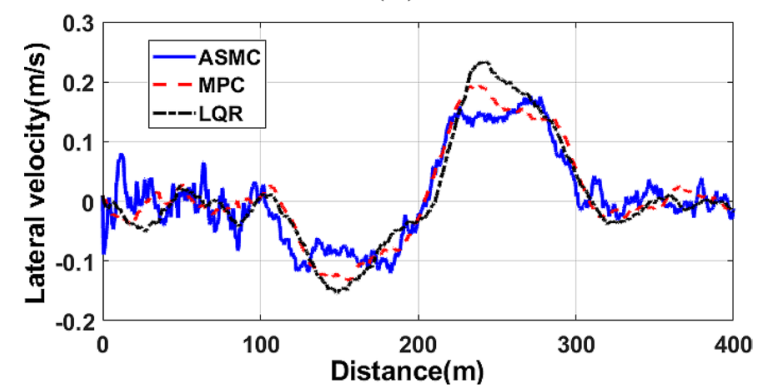

(c)

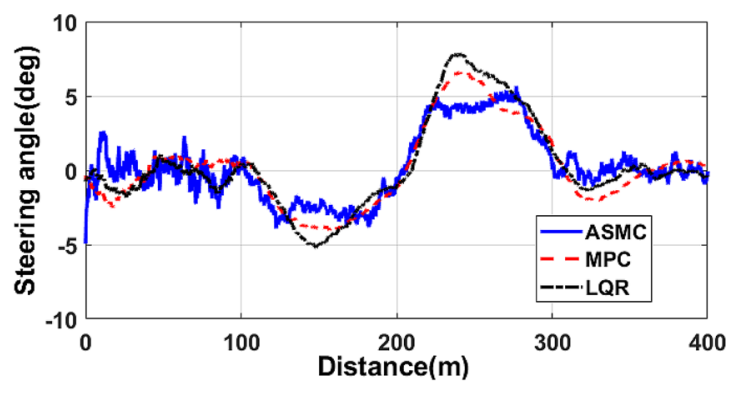

(d)

Figure 8 Vehicle states in experimental test I

Table 2 Error performance indexes in experimental test I

\begin{tabular}{lllll}
\hline Controller & $\boldsymbol{I}_{\text {IAE }}\left(\boldsymbol{y}_{L}\right)$ & $\boldsymbol{I}_{\text {ITAE }}\left(\boldsymbol{y}_{L}\right)$ & $\boldsymbol{I}_{\text {IAE }}\left(\boldsymbol{\varepsilon}_{\boldsymbol{L}}\right)$ & $\boldsymbol{I}_{\text {ITAE }}\left(\boldsymbol{\varepsilon}_{\boldsymbol{L}}\right)$ \\
\hline ASMC & 2.3 & 27.1 & 29.5 & 121 \\
MPC & 3.8 & 32.5 & 35.5 & 143 \\
LQR & 5.7 & 40.6 & 42.8 & 161 \\
\hline
\end{tabular}
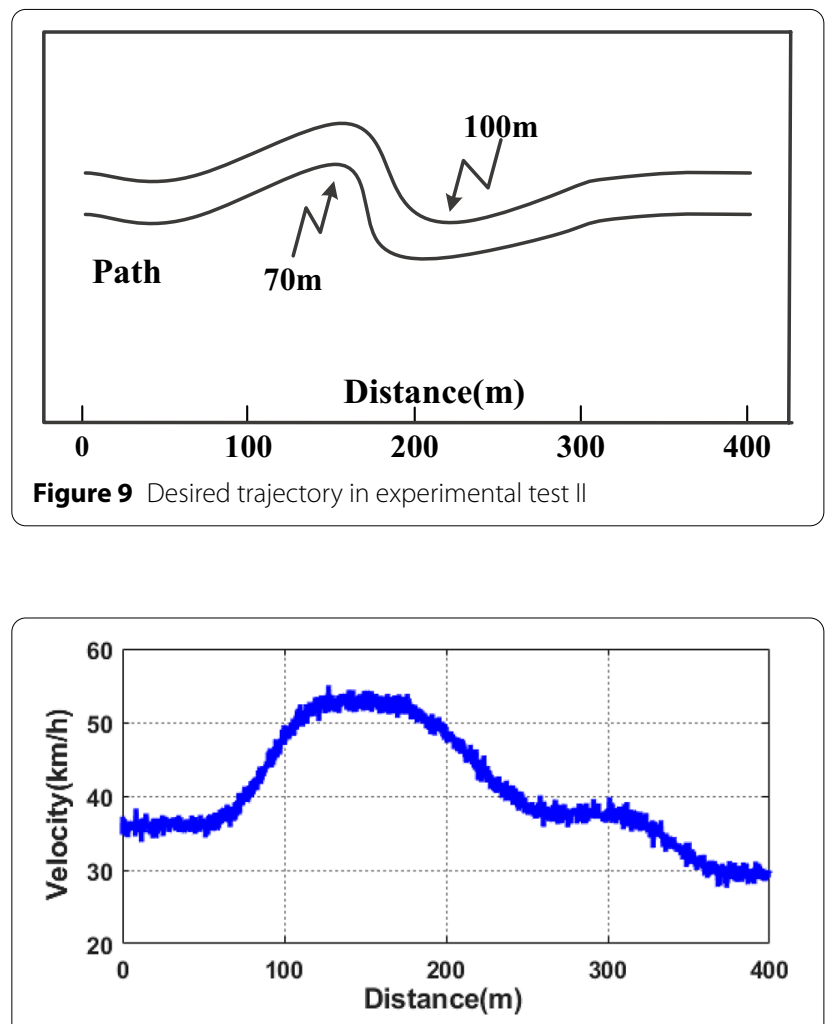

Figure 10 Longitudinal velocity in experimental test II

method, which can prouce the slight chattering phenomenon, as shown in Figure $8(\mathrm{~b}-\mathrm{d})$, but they are within an acceptable range.

Table 2 exhibits the error performance indexes of the three different control systems under the experimental test I, Table 2 shows that the values of IAE and ITAE controlled by proposed method is much smaller than the other two methods.

To further exhibit the tracking capability of the presented ASMC steering control strategy, the automatic steering maneuver of automated electric vehicle under the bad operation condition with the larger curvature is carried out. Figure 9 illustrates the desired path with maximum curvature of 0.014 . Figure 10 shows the varied velocity regulated by the longitudinal controller [35], and the initial velocity is $36 \mathrm{~km} / \mathrm{h}$. Besides, the initial lateral and angular errors are $-0.4 \mathrm{~m}$ and $3.5^{\circ}$.

The transient responses of automatic steering controller in experiment test II are exhibited in Figure 11. Figure 11(a) shows the maximum lateral displacement error managed by all the control approaches are occurred in the sector of largest curvature, the lateral errors can be restricted within $\pm 0.2 \mathrm{~m}, \pm 0.6 \mathrm{~m}$ and $\pm 1 \mathrm{~m}$ by the proposed ASMC method, the MPC control method and the LQR method. It is important to note that, the ASMC 


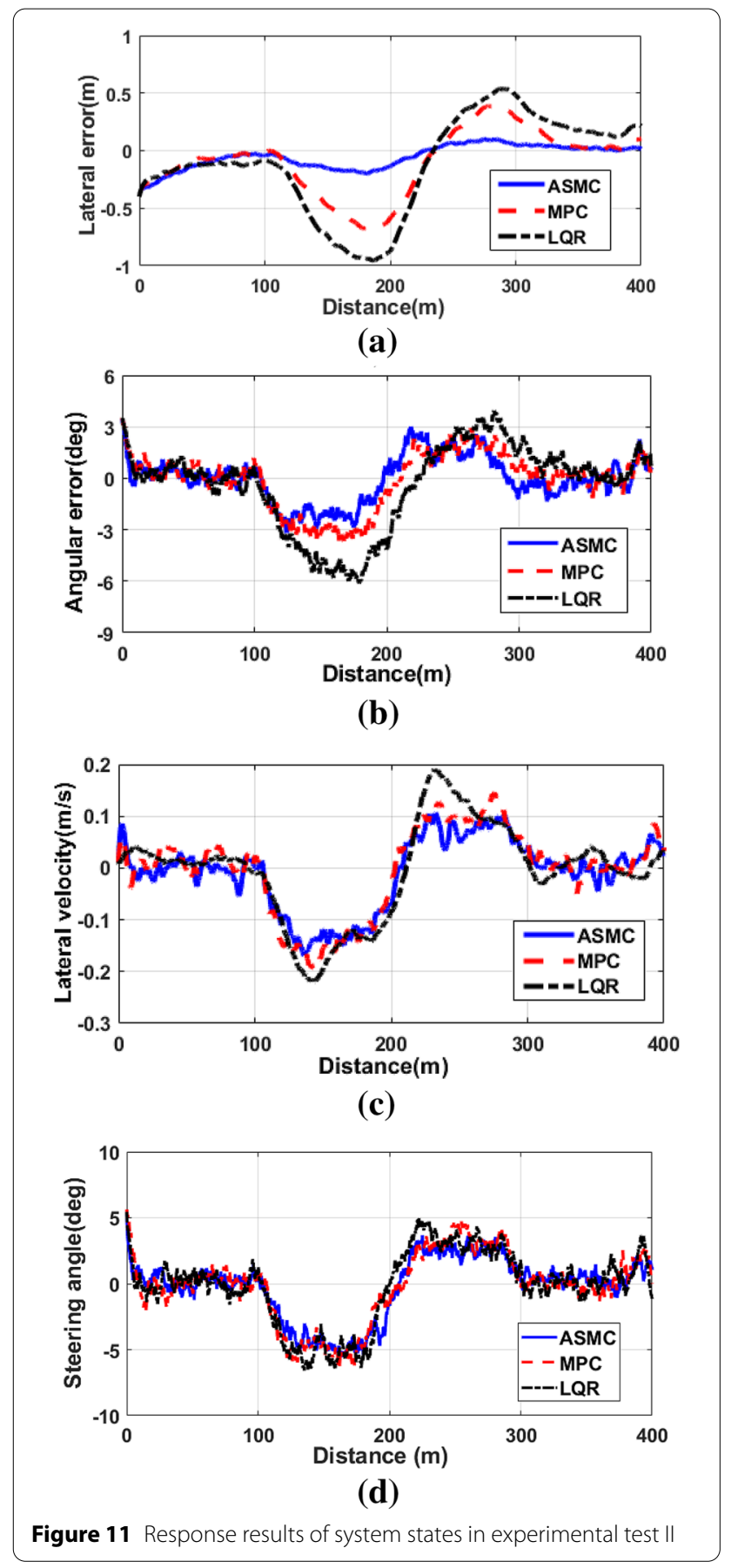

automatic steering control strategy improve the precision of lateral error greater than MPC controller and the LQR controller. Figure 11(b) demonstrates the response results of angular error, it illustrated that the presented ASMC control approach can limit the angular error within $\pm 2.8^{\circ}$, but the MPC controller and LQR controller merely guarantee the steady-state angular error bounded in $\pm 3^{\circ}$ and $\pm 6^{\circ}$, the presented control strategy has the
Table 3 Error performance indexes in experimental test II

\begin{tabular}{lllll}
\hline Controller & $\boldsymbol{I}_{\text {IAE }}\left(\boldsymbol{y}_{L}\right)$ & $\boldsymbol{I}_{\text {ITAE }}\left(\boldsymbol{y}_{L}\right)$ & $\boldsymbol{I}_{\text {IAE }}\left(\boldsymbol{\varepsilon}_{L}\right)$ & $\boldsymbol{I}_{\text {ITAE }}\left(\varepsilon_{L}\right)$ \\
\hline ASMC & 2.1 & 26.4 & 28.4 & 119 \\
MPC & 5.9 & 38.2 & 36.3 & 154 \\
LQR & 9.8 & 59.5 & 49.9 & 175 \\
\hline
\end{tabular}

advantages of higher accuracy than the other two control approaches. Figure 11(c) depicts the dynamic responses of lateral velocity, it is clear that all the approaches can ensure the lateral velocity be bounded within $\pm 0.2 \mathrm{~m} / \mathrm{s}$, however, the LQR control strategy has larger overshoot and oscillation than the ASMC control approach and the MPC control approach. Figure 11(d) demonstrates the corresponding front steering angle, it indicates that the chattering issue can be well handled by using the ASMC control strategy. The experimental results prove that the proposed automatic steering control strategy can well enhance the transient following performance of automated electric vehicle under the condition of varied velocity.

Table 3 shows the error performance indexes under the experimental test II, it can be observed that, the improvement in tracking accuracy of automatic steering control system can be achieved under the different road conditions by using the proposed control strategy.

\section{Conclusions}

1) The uncertain and time-varying model which accurately express the steering dynamic behaviors of unmanned electric vehicles are established, and in which the relationship between the look-ahead time and vehicle longitudinal velocity is revealed.

2) A neural-fuzzy-based adaptive sliding mode automatic steering control strategy of unmanned electric vehicles is presented to overcome the external disturbances and parametric uncertainties, which consists of an equivalent control law and a variable structure control law.

3) To effectively eliminate the chattering phenomenon, the control gains of variable structure control law is adaptively adjusted via the neural network technique, and the thickness of boundary layer is regulated by the fuzzy theory.

4) The stability of the closed-loop automatic steering control system is analyzed and proven. Furthermore, the results illustrate that the presented control strategy can effectively improve the trajectory following performances of automated electric vehicle. 


\section{Acknowledgements}

Authors express gratefulness for helpful comments from reviewer to improve this manuscript.

\section{Authors' contributions}

$J G$ was in charge of the whole trial; KL supervised the whole work of this paper; JF wrote the manuscript; YL assisted with sampling and laboratory analyses; JW investigated the experimental test. All authors read and approved the final manuscript.

\section{Authors' Information}

Jinghua Guo, born in 1982, is currently an associate professor at School of Aerospace Engineering, Xiamen University, China. He received his PhD degree from Dalian University of Technology, China, in 2012. He completed his post-doctoral fellowship at State Key Laboratory of Automotive Safety and Energy, Tsinghua University, China, in 2015. His research interests include vehicle engineering, intelligent vehicles, vehicle dynamics and control.

Keqiang Li, born in 1963, is currently a professor at State Key Laboratory of Automotive Safety and Energy, Tsinghua University, China. His research interests include Intelligent connected vehicles, vehicle dynamics and control.

Jingjing Fan, born in 1982, is currently an associate professor at School of Electrical and Control Engineering, North China University of Technology, China. He received his PhD degree from Tsinghua University, China, in 2009.

Yugong Luo, born in 1974, is currently a professor at State Key Laboratory of Automotive Safety and Energy, Tsinghua University, China. His research interests include Electric vehicles, vehicle dynamics and control.

Jingyao Wang, born in 1988, is currently an assistant professor at School of Aerospace Engineering, Xiamen University, China. She received his PhD degree from Peking University, China, in 2017. Her research interests include Multiagent system and networked control.

\section{Funding}

Supported by National Basic Research Project of China (Grant No. 2016YFB0100900), National Natural Science Foundation of China (Grant No. 61803319), Shenzhen Municipal Science and Technology Projects of China (Grant No. JCYJ20180306172720364), Fundamental Research Funds for the Central Universities of China (Grant No. 20720190015), State Key Laboratory of Automotive Safety and Energy of China (Grant No. KF2011).

\section{Competing interests}

The authors declare no competing financial interests.

\section{Author Details}

${ }^{1}$ School of Aerospace Engineering, Xiamen University, Xiamen 361005, China ${ }^{2}$ State Key Laboratory of Automotive Safety and Energy, Tsinghua University, Beijing 100084, China. ${ }^{3}$ School of Electrical and Control Engineering, North China University of Technology, Beijing 100144, China.

Received: 9 April 2020 Revised: 29 March 2021 Accepted: 11 August 2021 Published online: 18 September 2021

\section{References}

[1] J H Guo, K Q Li, Y G Luo. Coordinated control of autonomous four drive electric wheels for platooning and trajectory tracking using a hierarchical architecture. ASME Journal of Dynamic Systems Measurement \& Control, 2015, 137(10): 1-18.

[2] R Marino, S Scalzi, M Netto. Nest PID steering control for lane keeping in autonomous vehicles. Control Engineering Practice, 2011, 9(12): 1459-1467.

[3] E Kayacan, E Kayacan, H Ramon, et al. Towards agrobots: trajectory control of an autonomous tractor using type-2 fuzzy logic controllers. IEEE/ ASME Transactions on Mechatronics, 2015, 20(1): 287-293.

[4] S J Wu, H H Chiang, JW Perng, et al. The heterogeneous systems integration design and implementation for lane keeping on a vehicle. IEEE Transactions on Intelligent Transportation System, 2008, 9(2): 246-263.
[5] J H Guo, P Hu, L Li, R Wang. Design of automatic steering controller for trajectory tracking of unmanned vehicles using Genetic Algorithms. IEEE Transactions on Vehicular Technology, 2012, 61(7): 2913-2924.

[6] SThrun, M Montemerlo, H Dahlkamp, et al. Stanley: the robot that won the DARPA grand challenge. Journal of Field Robotics, 2006, 23(9): 661-692.

[7] HSTan, F Bu, B Bougler. A real-world application of lane-guidance technologies automated snowblower. IEEE Transactions on Intelligent Transportation System, 2007, 8(3): 538-548.

[8] J Perez, V Milanes, E Onieva. Cascade architecture for lateral control in autonomous vehicles. IEEE Transactions on Intelligent Transportation System, 2011, 12(1): 73-82.

[9] J Wang, M Ashour, C Lagoa, et al. A distributed traffic allocation algorithm for non-concave network utility maximization in connectionless communication networks. Automatica, 2019, 109: 1-11.

[10] J Huang, M Tomizuka. LTV controller design for vehicle lateral control under fault in rear sensors. IEEE/ASME Transactions on Mechatronics, 2005, 10(1): 1-7.

[11] P Falcone, F Borrelli, J Asgari, et al. Predictive active steering control for autonomous vehicle systems. IEEE Transactions on Control Systems Technology, 2007, 15(2): 566-580.

[12] E Kayacan, E Kayacan, H Ramon, et al. Towards agrobots: identification of the yaw dynamics and trajectory tracking of an autonomous tractor. Computers and Electronics in Agriculture, 2015, 115: 78-87.

[13] J H Guo, Y G Luo, K Q Li, et al. Coordinated path-following and direct yaw-moment control of autonomous electric vehicles with sideslip angle estimation. Mechanical Systems and Signal Processing, 2018, 105: 183-199.

[14] N M Enache, S Mammar, M Netto, et al. Driver steering assistance for lane-departure avoidance based on hybrid automata and composite Lyapunov function. IEEE Transactions on Intelligent Transportation System, 2010, 1(1): 28-39.

[15] J H Guo, L Li, K Li, et al. An adaptive fuzzy-sliding lateral control strategy of automated vehicles based on vision navigation. Vehicle System Dynamics, 2013, 51(10): 1502-1517.

[16] H Li, J Yu, C Hilton, et al. Adaptive sliding mode control for nonlinear active suspension vehicle systems using T-S Fuzzy Approach. IEEE Transactions on Industrial Electronics, 2013, 60(8): 3328-3338.

[17] M Kim, H Joe, J Kim, et al. Integral sliding mode controller for precise manoeuvring of autonomous underwater vehicle in the presence of unknown environmental disturbances. International Journal of Control, 2015, 88(10): 2055-2065

[18] R Pradhan, B Subudhi. Double integral sliding mode MPPT control of a photovoltaic system. IEEE Transactions on Control Systems Technology, 2016, 24(1): 285-292.

[19] A Oveisi, T Nestorovic. Robust observer-based adaptive fuzzy sliding mode controller. Mechanical Systems and Signal Processing, 2016, 76(77): 58-71.

[20] R Vrabel. On the approximation of the boundary layers for the controllability problem of nonlinear singularly perturbed systems. Systems \& Control Letters, 2012, 61(3): 422-426.

[21] M Khazaee, A Markazi, S Rizi, et al. Adaptive fuzzy sliding mode control of input-delayed uncertain nonlinear systems through output-feedback. Nonlinear Dynamics, 2017, 87(3): 1943-1956.

[22] A F Amer, E A Sallam, W M Elawady. Adaptive fuzzy sliding mode control using supervisory fuzzy control for a 3DOF planar robot manipulators. Applied Soft Computing, 2011, 11(8): 4943-4953.

[23] W M Bessa, M S Dutra, E Kreuzer. An adaptive fuzzy sliding mode controller for remotely operated underwater vehicles. Robotics and Autonomous Systems, 2010, 58(1): 16-26.

[24] H Li, J Wang, H K Lam, et al. Adaptive sliding mode control for interval type-2 fuzzy system. IEEE Transactions on Systems, Man, and Cybernetics: Systems, 2016, 46(12): 1654-1663.

[25] R J Wai, R Muthusamy. Fuzzy neural network inherited sliding mode control for robot manipulator including actuator dynamics. IEEE Transactions on Neural Networks and Learning Systems, 2013, 24(2): 274-287.

[26] M Rahmani, A Ghanbari, M M Ettefagh. Hybrid neural network fraction integral terminal sliding mode control of an Inchworm robot manipulator. Mechanical Systems and Signal Processing, 2016, 80: 117-136.

[27] M Mitschke, H Wallentowitz. Dynamik der Kraftfahrzeuge. Berlin: Springer, 2004. (in Germany) 
[28] R Rajamani. Vehicle dynamics and control. Berlin: Springer, 2012.

[29] J H Guo, Y G Luo, K Li. Robust gain-scheduling automatic steering control of unmanned ground vehicles under velocity-varying motion, Vehicle System Dynamics, 2019, 57(4): 595-616.

[30] K Li, T Cao, Y Luo, et al. Intelligent environment friendly vehicles: concept and case studies, IEEE Transactions on Intelligent Transportation System, 2012, 3(1): 318-328

[31] J Wang, G Wen, Z Duan, et al. Distributed stochastic consensus control integrated with performance improvement: A consensus-regionbased approach. IEEE Transactions on Industrial Electronics, 2020, 67(4): 3000-3012.

[32] VT Yen, Y N Wang, P V Cuong. Recurrent fuzzy wavelet neural networks based on robust adaptive sliding mode control for industrial robot manipulators. Neural Computing \& Applications, 2018, 18: 1-14.

[33] H Leung, T Lo, S Wang. Prediction of noisy chaotic time series using an optimal radial basis function neural network. IEEE Transactions on Neural Networks, 2001, 12(5): 1163-1172.

[34] A Saghafinia, HW Ping, M N Uddin, et al. Adaptive fuzzy sliding mode control into chattering-free IM drive. IEEE Transactions on Industry Applications, 2015, 51(1): 692-701.
[35] J H Guo, Y G Luo, K Q Li. A novel fuzzy-sliding automatic speed control of intelligent vehicles with adaptive boundary layer. International Journal of Vehicle Design, 2017, 73(4): 300-318.

[36] J J Slotine, T S Liu. Applied nonlinear control. Englewood Cliffs: Prentice Hall, 1991.

[37] J H Guo, Y Luo, K Li. An adaptive hierarchical trajectory following control approach of autonomous four-wheel independently drive vehicles. IEEE Transactions on Intelligent Transportation System, 2018, 19(8): 2482-2492.

[38] J H Guo, J Y Wang, Y Luo, et al. Robust lateral control of autonomous fourwheel independent drive electric vehicles considering the roll effects and actuator faults. Mechanical Systems and Signal Processing, 2020, 143: 106773

[39] J H Guo, J Y Wang, Y Luo, et al. Takagi-Sugeno fuzzy-based robust $H \infty$ integrated lane-keeping and direct yaw moment controller of unmanned electric vehicles. IEEE/ASME Transactions on Mechatronics, 2021, doi: https://doi.org/10.1109/TMECH.2020.3032998.

\section{Submit your manuscript to a SpringerOpen ${ }^{\circ}$ journal and benefit from:}

- Convenient online submission

- Rigorous peer review

- Open access: articles freely available online

- High visibility within the field

- Retaining the copyright to your article

Submit your next manuscript at $\boldsymbol{\Delta}$ springeropen.com 